\title{
Associative and perceptual dominance in compounding stimuli
}

\author{
ZAKHOUR I. YOUSSEF AND MICHAEL DOSEY, \\ DEPARTMENT OF PSYCHOLOGY, EASTERN MICHIGAN \\ UNIVERSITY, Ypsilanti, Mich. 48197
}

After learning verbal paired-associate $(P-A)$ lists with single stimuli, 90 college Ss were tested on $(P-A)$ lists with either single or compound stimuli. Compounds which consisted of two relevant cues were not superior to a single relevant cue while each of these conditions was superior to compounds consisting of one relevant and one irrelevant cue, particularly when the irrelevant cue was perceptually dominant. Associative and perceptual factors interacted in determining the transfer effects of compounding.

Previous research on compound stimuli in verbal P-A learning has led to conflicting results. Weiss \& Margolis (1954), Hill \& Wickens (1962), Feldman (1963), and Saltz (1963) found that compounding cues enhances P-A learning while Horowitz, Lippman, Norman, \& McCronkie (1964) and Underwood, Ham, \& Ekstrand (1962) reached the opposite conclusion. Such contradiction in results have been ascribed to the variable level of stimulus complexity employed in these studies. To mitigate against the presumable inherent interaction of cue complexity and compounding in P-A learning, this study employed a transfer design to investigate compounding effects after associative connections had been formed with single cues. Applying Pavlovian conditioning principles (Pavlov, 1927) to verlal learning phenomena, it is hypothesized: (1) After an initial training period with pairs in which the stimuli are single cues, transfer of training to a compound stimulus composed of two relevant cues would be superior to a single stimulus (à la Pavlov's summation paradigm); (2) Transfer to a single relevant stimulus, however, would be superior to a compound composed of one relevant and one irrelevant cue (à la Pavlov's paradigm of external inhibition); (3) Decrement in transfer due to an irrelevant component cue is directly related to the spatial proximity of that cue to the response (perceptual dominance effects analogous to Pavlov's concept of stimulus intensity).

Method. Ninety college sophomores learned two P-A lists, 10 trials on a Practice List (PL) and four trials on Test List (TL). PL consisted of 12 pairs: two sets of stimuli paired, one at a time, to a set of six responses. TL consisted of six pairs. The responses of $P L$ and TL were identical for all groups. The TL stimuli varied as follows: For Group A (single relevant cue), the stimuli were only one of the two sets used in PL. For Group B (two relevant cues), the two relevant stimuli in PL were compounded in TL by placing them next to each other separated only by a comma. To control for pre-experimental associative differences between the two sets of stimuli, spatial position of the two relevant cues was counterbalanced. For Group C (relevant proximal + irrelevant distal), the stimuli were also compounds but only the cue spatially proximal to the response side was relevant. Group $\mathbf{C}^{\prime}$ (relevant distal + irrelevant proximal) differed from Group $C$ only in spatial position of the irrelevant cue which was to the left of the relevant one for Group $\mathrm{C}$ and to the right of it for $\mathrm{C}^{\prime}$. Thus shift of position presumably augments the perceptual dominance of the irrelevant cue for $C^{\prime}$. On the basis of the three hypotheses above, the TL relative performance of the four groups was predicted to be: $\mathrm{B}>\mathrm{A}>\mathrm{C}>\mathrm{C}^{\prime}$.

All items employed were $50-90 \%$ glaze CVC nonsense syllables. They appeared on a Lafayette memory drum with $2 \mathrm{sec}$ intra- and $6 \mathrm{sec}$ intertrial intervals. The intratrial interval was extended to $4 \mathrm{sec}$ only for TL. Standard $\mathrm{P}$-A instructions were given to all Ss who were randomly assigned to each of the four groups with the restriciton of 20 Ss in each of $A, C$, and $C^{\prime}$ Groups and 30 in the B group. All Ss were instructed to pronounce aloud the stimuli, whether compound or single, and responses during TL learning and test trials. In both PL and TL, learning and test trials alternated. Six different random orders of presentation were used to reduce serial position effects.

Results. Table 1 presents the means and standard deviations of total number correct on $10 \mathrm{PL}$ trials and the first two TL trials and the adjusted means on the latter measure. Since using adjusted means reduces error variance, a series of $t$ tests was conducted on the TL adjusted means with the following results: Contrary to
Table 1

Means and Standard Deviations of Total Correct Responses on 10 PL Trials and First Two TL Trials

\begin{tabular}{lrcccc} 
& & \multicolumn{4}{c}{ Group } \\
\cline { 3 - 6 } List & & A & B & C & C' \\
\hline \multirow{4}{*}{ PL } & $\bar{X}$ & 61.05 & 46.37 & 54.85 & 49.00 \\
& SD & 23.17 & 21.02 & 22.86 & 19.52 \\
& $\mathrm{~N}$ & 20 & 30 & 20 & 20 \\
& $\bar{X}$ & 10.35 & 8.58 & 7.35 & 4.05 \\
TL & SD & 2.49 & 2.34 & 2.11 & 2.03 \\
Adjusted & $\bar{X}$ & 9.50 & 9.02 & 7.09 & 4.34 \\
\hline
\end{tabular}

prediction, Groups A and B did not differ significantly from each other $(p>20)$. Thus the first hypothesis that transfer to a compound stimulus composed of two relevant cues would be superior to a single relevant stimulus (summation effects) was not supported. However, as predicted, both Groups A and B were superior to each of $C$ and $C^{\prime}$ (for each comparison, $t \geqslant 3.0, d f=$ $38, p<.01$ ) supporting the second hypothesis that a compound of one relevant and one irrelevant cue (external inhibition effects) would hinder transfer in P-A learning whether such compounding should be contrasted with a single relevant cue or with a compound composed of two relevant cues. Also as predicted, Group $C$ was significantly superior to Group $C^{\prime}(t=5.01$, $d f=38$, $\mathrm{p}<.001)$ supporting the third hypothesis that decrement in transfer due to an irrelevant component cue is directly related to the spatial proximity of that cue to the response (perceptual dominance or intensity effects).

Analyses performed on total correct responses over three and four TL trials as well as on intrusion data yielded similar results.

Discussion. The findings support the applicability of the Pavlovian principles of external inhibition and stimulus intensity to compounding effects in P-A learning. Summation effects, however, did not obtain by compounding two relevant cues. Perhaps, two factors masked summation effects: (a) generalization decrement, and (b) insufficient intratrial interval to enable Ss to utilize both relevant cues. Concerning the first, it is obvious that stimulus similarity between PL and TL was higher for Group A than for B. Thus a more pronounced generalization decrement for Group B may have neutralized possible gains contributed by an additional relevant cue. In fact, the four TL means show a perfect generalization decrement gradient as stimulus similarity between $\mathrm{TL}$ and $\mathrm{PL}$ decreases. Present findings clearly demonstrate generalization decrement effects on transfer and suggest generalization as a possible mechanism underlying external inhibition and perceptual dominance effects. Concerning the second, an intratrial interval of 4 sec may not allow Ss to utilize more than one cue. If so, stimulus items in Groups A and $B$ would have been functionally equivalent. Attending only to one cue would be sufficient to effect the transfer decrement obtained for Groups $\mathrm{C}$ and $C^{\prime}$ since that cue may be the irrelevant one.

\section{REFERENCES}

FELDMAN, S. E. Probalistic hierarchies to ambiguous concept classes. J. exp. Psychol., 1963, 65, 240-247.

HILL, F. A., \& WICKENS, D. D. The effect of stimulus compounding in paired-associate learning. J. verbal Learn. verbal Behav., 1962, 1, 144-151. HOROWITZ, H. M., LIPPMAN. L. G., NORMAN, S. A., \& MicCRONKIE, G. W. Compund stimuli in paired-associate learning. J. exp. Psychol., 1964. 67, 132-141.

PAVLOV, I. P. Conditioned reflexes. (Translated by G. V. Anrep), London: Oxford Univ. Press, 1927.

SALTZ, E. Compound stimuli in verbal learning: Cognitive \& sensory differentiation vs stimulus selection. J. exp. Psychol, 1963, 66, 1-5.

UNDERWOOD, B. J., HAM, M., \& EKSTRAND, B. Cue selection in paired associate learning. J. exp. Psychol., 1962, 64, 405-409.

WEISS, W., \& MARGOLIS, G. The effect of content stimuli on learning and retention. J. exp. Psychol., 1954, 48, 318-322. 